\title{
CÓMO SE CREA UNA CÁTEDRA: HIPÓLITO RODRÍGUEZ PINILLA Y LA HIDROLOGÍA MÉDICA ESPAÑOLA
}

\author{
Dr. D. Juan Antonio Rodríguez Sánchez \\ Historia de la Ciencia. Universidad de Salamanca. \\ jarshm@usal.es
}

\section{Introducción}

En 1913 se creó en la Universidad Central de Madrid la primera cátedra de Hidrología Médica existente en España, aunque reducida en sus competencias al ámbito del doctorado. El primer profesor en desempeñar el cargo de catedrático fue el salmantino Hipólito Rodríguez Bartolomé, al que se le conocería por los apellidos paternos de Rodríguez Pinilla. Desde ese privilegiado puesto ejerció una influencia decisiva en los cambios que vivió la especialidad en las tres décadas posteriores a su nombramiento. Este artículo pretende sintetizar los asuntos clave para la comprensión de los tradicionales avatares que han acompañado a esta disciplina en España.

\section{2. ¿Por qué una cátedra?}

La Universidad española de principios del siglo XX no era más propensa que la que ahora conocemos a crear nuevas cátedras. Resulta indudable que la ciencia de la época experimentaba una profunda transformación y que los profesionales de la medicina españoles comenzaban a beneficiarse de una formación ampliada en el extranjero que les capacitaba en novedosas áreas de la investigación biomédica. Sin embargo, el reconocimiento oficial de una nueva disciplina a través de su autonomía en el conjunto de las enseñanzas no dependía exclusivamente de sus avances y nuevos descubrimientos. Que la Hidrología Médica alcanzase ese estatus académico plantea consideraciones sobre los posibles motivos para crear una cátedra.

\subsection{Motivos sociales}

Resulta una justificación clásica a cualquier decisión el que se fundamente en el bien común. En este caso el de las personas usuarias de los balnearios españoles. En 1901 no llegaban éstas al 0,47\% del total de la población española, siendo una cuarta parte calificada como pobre de solemnidad. Este número había caído desde las cifras récord de 1883 y en la década que precedió a la creación de la cátedra se perdieron 18.000 agüistas hasta quedar reducidos a menos de setenta mil. Si las causas se encontraban en las fraudulentas estadísticas, en los balnearios ilegales, en la competencia de los baños de mar o en el éxodo de los bañistas "de calidad" a centros termales extranjeros fueron debates propios de la época. Desde esta perspectiva se buscó como una de las posibles justificaciones para la creación de la cátedra su potencial papel en el estímulo de la industria balnearia nacional.

\subsection{Motivos académicos}

Las aguas mineromedicinales eran estudiadas como un remedio más en la asignatura de Materia Médica y, desde 1866, incluso figuraba su nombre en la de "Ampliación de la Terapéutica y Farmacología. Hidrología Médica". Sin embargo, los conocimientos que se podían adquirir eran tan escasos que cualquier formación para el ejercicio en un balneario (y, claro está, para superar las oposiciones precisas para ello) había de pasar por el aprendizaje autodidacta. Los defensores de la incorporación de una asignatura dedicada en exclusiva a la Hidrología Médica, argumentaban que el desconocimiento de las aguas mineromedicinales impedía que los médicos derivasen a sus pacientes a los balnearios y que aquí radicaba uno de los principales problemas del termalismo español: la falta de cultura hidrológica tanto entre los propios profesionales de la salud como entre las personas que les consultaban.

\subsection{Motivos profesionales}

\subsubsection{El Cuerpo de Médicos de Baños}

Creado, con asombrosa precocidad en España, en el año 1816 y unificado oficialmente en escalafón en el año 
1877, a él se accedía por oposición y era el que regulaba el acceso a los diferentes balnearios. Las prebendas de las que en algún momento gozaron hicieron que la pertenencia a esta corporación fuera muy codiciada por cualquier médico, sin tener en cuenta la gran diferencia entre las ganancias que se podían obtener en los grandes balnearios frente a las escasísimas de los de orden inferior. Dado el sistema de escalafón de antigüedad, éstos últimos eran ocupados por los profesionales más bisoños, quienes, aunque fuesen brillantes, habrían de esperar la jubilación o el fallecimiento de sus predecesores para optar a una plaza de Médico-Director de un balneario de prestigio con suficientes agüistas como para asegurar unos buenos emolumentos.

Esta situación provocó divisiones, continuos enfrentamientos y reformas superficiales del Cuerpo que no resolvían sus problemas: en 1887 surgió la figura de los Médicos-Directores Supernumerarios, en 1890 el Cuerpo de Médicos-Directores de Aguas MineroMedicinales en Ultramar y en 1903 aparecieron los Médicos Habilitados. Figuras que, por cumplir los mismos requisitos que los médicos del Cuerpo, exigieron los mismos derechos y acabaron integrados en un único y conflictivo escalafón de antigüedad.

Fueron los Médicos Habilitados, encabezados por Rosendo Castells Ballespí, quienes constituyeron una Asamblea de Médicos de Baños desde la que se comenzó a reclamar la creación de una cátedra, entre las de licenciatura, para la enseñanza de la Hidrología Médica.

\subsubsection{Sociedad Española de Hidrología Médica}

Aunque habían existido intentos previos (en 1848, 1854, 1861 e, incluso, por la comisión del frustrado Reglamento de 1856) de crear una sociedad científica, ésta no surgió hasta 1877, al amparo del Reglamento de 1874. Buscaba cohesión de los profesionales hidrólogos en lo científico y lo social, la unidad en la defensa de intereses comunes y la promoción de la investigación y la docencia. Su más significativa y perdurable contribución a ese proyecto fue la creación de los Anales, una revista que, con cambios e intermitencias, ha perdurado como seña identitaria. Los enfrentamientos dentro del Cuerpo se trasladaron bien pronto a la Sociedad, por la que no se sentían representados los médicos zagueros del escalafón, teniendo como resultado que al finalizar el siglo XIX desapareciesen los Anales, el Anuario Oficial de las Aguas Minerales de España y la sociedad perdiese sus locales. Una cátedra, el garante de un entorno académico presumiblemente independiente, se ofreció como una forma de regeneración corporativa.

\subsubsection{Médicos generales}

Si un médico, debidamente titulado, tenía reconocida su capacidad para recetar cualquier fármaco, que los Médicos

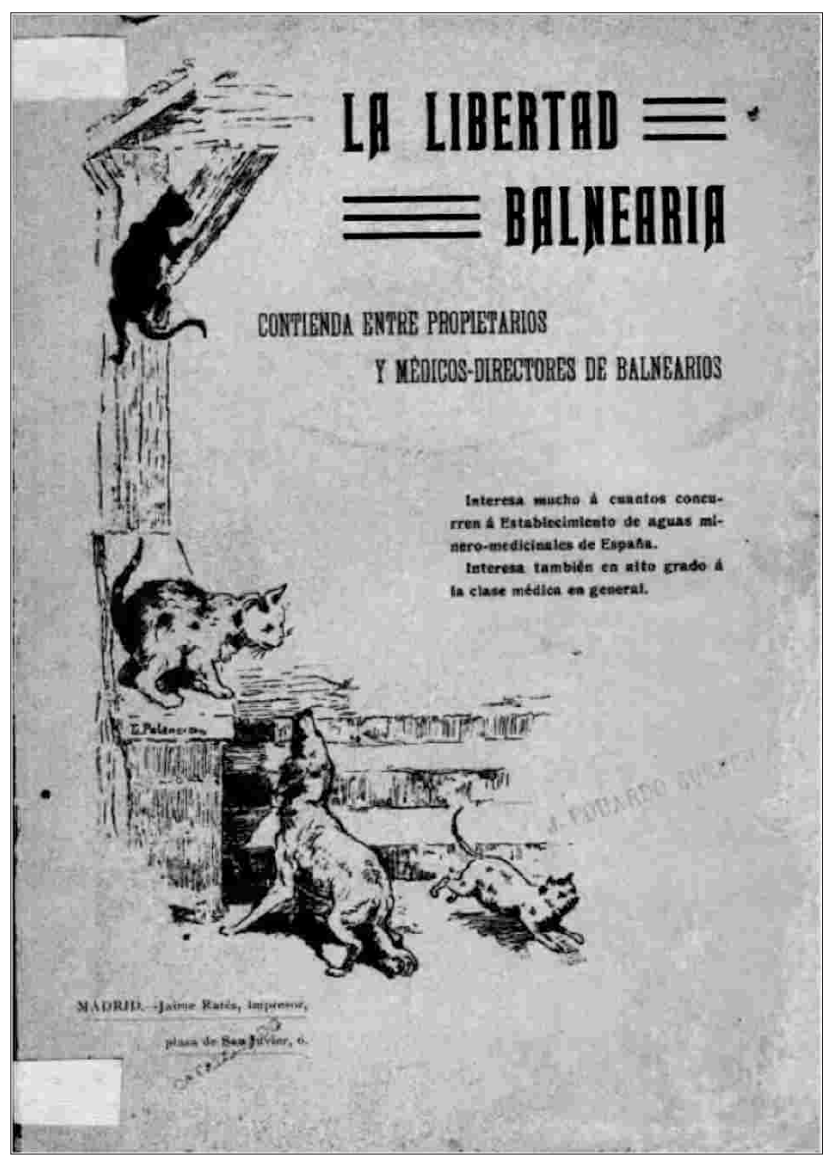

Fig. 1. La libertad balnearia (1910).

de Baños hubiesen monopolizado el remedio hidromineral era considerado como una vulneración de los derechos del resto de los profesionales de la medicina. El reconocimiento en 1868 de la figura del médico de la localidad como Médico Libre o la del Médico-Director Interino, en los casos en los que ningún médico del Cuerpo quisiera acudir al balneario, fueron intentos tímidos de mediar en el conflicto. La cátedra ofrecía en este caso una de las vías más interesantes y, como se demostró a la larga, más revulsiva: la formación académica en Hidrología Médica permitiría la contratación, en caso de la ausencia de miembros del Cuerpo, de cualquier profesional que acreditase estudios de la asignatura.

\subsection{Motivos empresariales}

El balneario, como empresa, ofrecía una singularidad generadora de continuas tensiones. Pese a tratarse de negocios habitualmente privados, el hecho de ser el agua mineromedicinal un bien de utilidad pública llevó a que fuera ese Cuerpo de Médicos de Baños reconocido oficialmente el que determinase qué profesional había de dirigir cada balneario, sin que en esta decisión pudiesen 


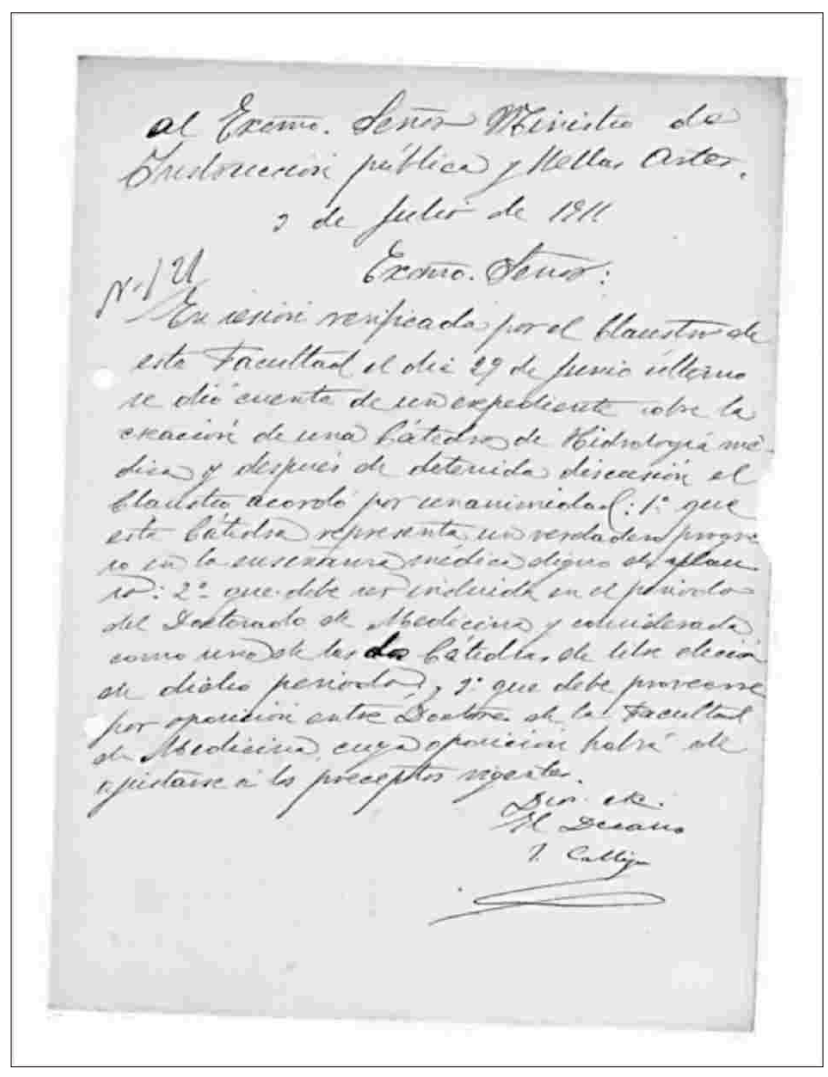

Fig. 2. Escrito de solicitud de creación de cátedra, de 2-71911 (AFMUC).

participar los propietarios de las instalaciones. Cada temporada podía suponer un nuevo reto con enfrentamientos entre un empresario que evaluaba minuciosamente la rentabilidad de sus inversiones y un médico que, en el ejercicio de su dirección, podía introducir sorprendentes criterios para administrar las aguas.

Con el nombre de "libertad balnearia" los propietarios de esos establecimientos exigían poder elegir al profesional que había de dirigir el balneario (Fig. 1). La aparición de los Médicos Habilitados pretendía responder a su demanda, pero, en número tan poco significativo, que el descontento no cedió. Una vez más entró en juego el papel que podía jugar una cátedra para ofrecer un amplio abanico de médicos formados en Hidrología Médica entre los que los empresarios pudiesen encontrar al director idóneo.

\section{3. ¿Cómo se crea una cátedra?}

Lo expuesto anteriormente exhibe unos motivos bastante débiles para la creación de una cátedra de Hidrología
Médica si los contraponemos con la realidad existente: una terapéutica con una demanda social no sólo escasa sino en clara disminución, unos profesionales que no pasaban del centenar y muy mal avenidos y una investigación ausente tanto de los balnearios como de la academia. Sin embargo, se iba a crear en Madrid una de las primeras cátedras universitarias de la especialidad en Europa, casi una década antes de que la International Society of Medical Hydrology and Climatology comenzase a reclamarla.

\subsection{Demanda y visibilización}

El descontento y la crítica de los médicos que se agrupaban en torno a la Revista Médico-Hidrológica Española (los Médicos Habilitados) fueron trascendentales para visibilizar la demanda de una enseñanza obligatoria de la Hidrología Médica en los estudios de Medicina. De la referida Asamblea de 1905 partió la primera solicitud, dirigida al Ministro de Instrucción Pública, de creación de una cátedra.

La Revista no sólo actuaba de portavoz sino que generó asiduamente noticias y artículos que mostraban, mediante la exposición de la marcha de los estudios en otros países, que la demanda era internacional y que contaba con el respaldo de los congresos internacionales de la disciplina. Cuando Manuel Márquez accedió a la cátedra de Terapéutica, una entrevista y una oportuna pregunta permitieron recoger lo que había de ser el proyecto de creación de la cátedra ${ }^{1}$.

También en esto se mostró menos vital la Sociedad, pues hasta el 17 de abril de 2011 no presentó una solicitud de creación de cátedra. El 29 de junio del mismo año había de hacerlo el claustro de la Facultad de Medicina de Madrid y el dos de julio se elevaba al Ministerio de Instrucción Pública la petición de una cátedra de Hidrología Médica como de libre elección del doctorado (Fig. 2).

\subsection{Oportunidad}

Aunque es indudable que la movilización de los Médicos Habilitados creaba un ambiente propicio, existió una figura clave sin la que no se podría explicar este cambio. La citada Asamblea de Médicos de Baños fue presidida por un político de talante liberal y progresista que, además de ser médico, era Médico de Baños, tercero en el escalafón y director del balneario de Cestona. Se trataba de Amalio Gimeno y Cabañas (Fig. 3). Fue él quien vio en la cátedra la posibilidad de regenerar el Cuerpo, la Sociedad y la industria termal española en un ambicioso

1 Marquez, M. consideraciones acerca de la cura hidromineral y su enseñanza. Revista Médico-Hidrológica Española. 1909, 10:356-357. 


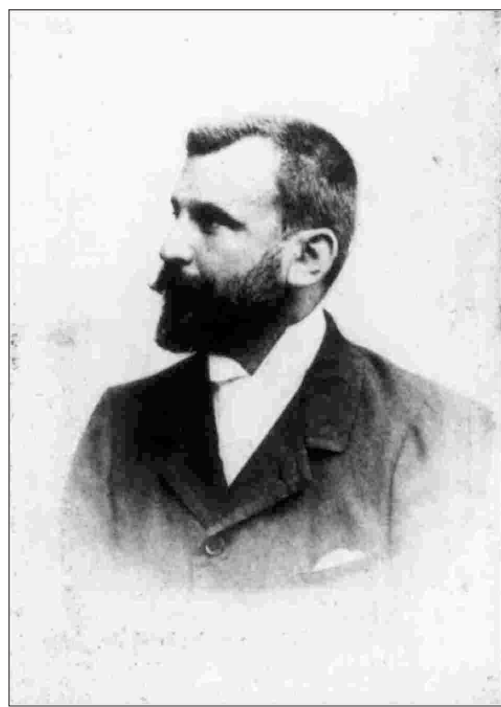

Fig. 3. Amalio Gimeno y Cabañas.

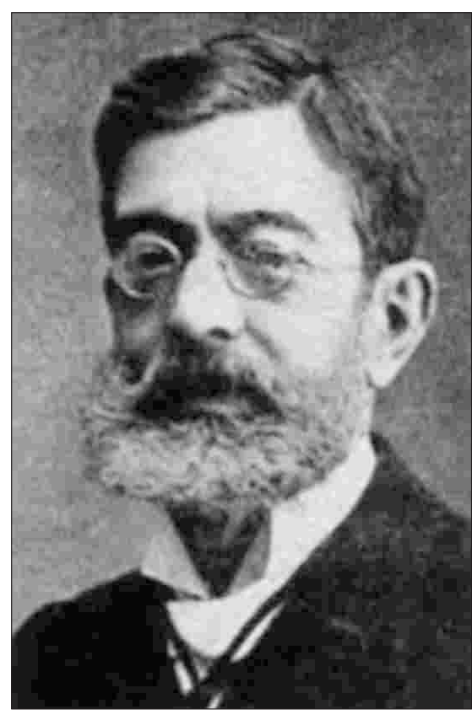

Fig. 4. Hipólito Rodríguez Pinilla. intento de europeización que colocase a España como pionera de ese movimiento dignificador de la ciencia hidrológica. Desde la Junta de Ampliación de Estudios estimuló esta comunicación internacional para la Hidrología y medió para traer a España el IX Congreso Internacional de Hidrología Médica, del que fue nombrado presidente, que había de celebrarse en 1912, aunque acabó posponiéndose al año siguiente.

En este escenario idóneo, desde su segundo mandato al frente del Ministerio de Instrucción Pública, estimuló en 1911, durante una cena aniversario de la Sociedad Española de Hidrología Médica, a que sus compañeros le solicitasen formalmente crear la cátedra. De esta forma, al escenario incorporó los actores y sólo faltaba acabar de perfilar el libreto.

\subsection{Legislación}

Un Real Decreto de cinco de enero de 1912 creaba una cátedra de Hidrología Médica entre las del doctorado y, por tanto, en la Universidad de Madrid. Rosendo Castells criticó la coincidencia de una oposición en las mismas fechas que el Congreso Internacional, del que era secretario, pues consideraba que condicionaría al tribunal a no dejar la plaza desierta.

Ese tribunal fue nombrado por una Real Orden de siete de marzo de 1912 y tenía al Decano de la Facultad de Medicina, Julián Calleja, como presidente y al anteriormente mencionado Manuel Márquez como secretario. Para las tres vocalías restantes fueron nombrados Taboada, Alonso Sañudo y Manzaneque. Los suplentes eran Martín Salazar, Pinilla, Cerrada y Gurucharri. Sin embargo, sólo uno de los miembros de ese tribunal inicial llegó al final de la oposición.

\subsection{Realización}

La enfermedad de Alonso Sañudo, de la que al poco tiempo fallecería, le llevó a renunciar a su vocalía. Lo mismo hicieron Manzaneque y Pinilla pues se presentaban como candidatos en la oposición. De este modo, al constituirse el tribunal en enero de 1913 las vocalías las ocupaban Taboada, Martín Salazar y Gurucharri. Para el mes de mayo habían fallecido otros dos miembros, Taboada y el presidente Calleja, por lo que el tribunal se compuso de sólo tres personas.

De los catorce candidatos iniciales sólo concursaron cuatro: los ya conocidos Pinilla y Manzaneque, médicos del Cuerpo de Baños, y Enrique Fernández Sanz y Adolfo Hinojar Pons, que eran Médicos Habilitados. Tras los ejercicios, Hinojar fue el primero relegado y la segunda votación repartió los apoyos por igual: Márquez votó a Fernández Sanz, Gurucharri a Manzaneque y Martín Salazar a Pinilla. En la tercera hubo unanimidad para designar como catedrático a Hipólito Rodríguez Pinilla y Bartolomé (Fig. 4).

\section{Hipólito Rodríguez Pinilla}

\subsection{Médico y ecléctico}

Hipólito Rodríguez Bartolomé nació en Salamanca el trece de agosto de 1860. Su madre, María Concepción, era de Ledesma, una localidad vinculada a unos afamados baños que condicionaron en buena medida su carrera profesional.

Estudió Medicina en Madrid, donde se licenció en 1881 y se doctoró al año siguiente. Heredero de las postrimerías del escepticismo terapéutico, comenzó su andadura clínica 


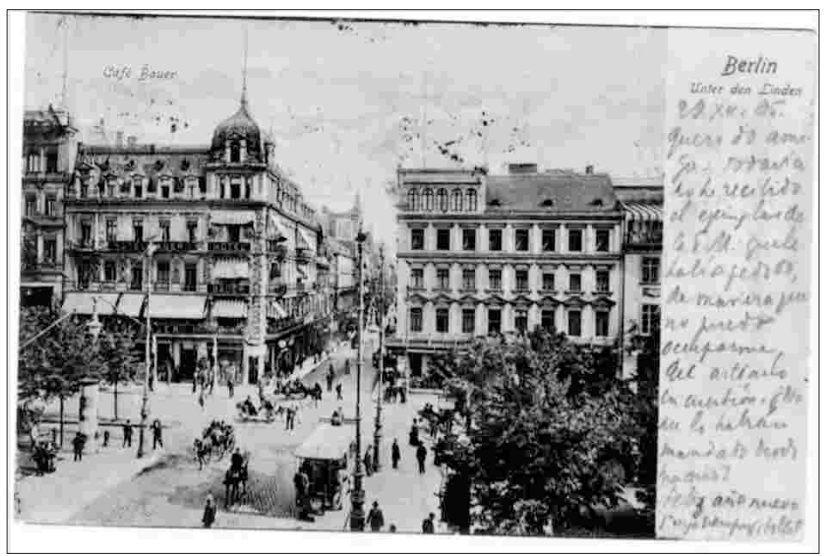

Fig. 5. Postal enviada desde Berlín a Miguel de Unamuno (CMU, 38/37, 13).

en el terreno de una disciplina que gozaba en esos momentos de gran atención por parte de los médicos y de las altas esferas sociales: la homeopatía. Fue alumno y, más tarde, profesor del Instituto Homeopático de Madrid y médico del Hospital Homeopático de San José, así como director de la revista El Criterio Médico. En esta trayectoria coincidió, y en muchos aspectos, con Anastasio García López: médico, hidrólogo, homeópata y catedrático de la Universidad de Salamanca.

En 1901 era profesor auxiliar en la Facultad Municipal de Medicina de la Universidad Literaria de Salamanca y, ese mismo año, pasó a ocupar como interino la cátedra de Patología Médica. El reconocimiento estatal de la Facultad de Medicina de Salamanca le permitió ser uno de los primeros catedráticos oficiales de la misma al ganar la oposición a la de Enfermedades de la Infancia en 1902. En esas fechas tenía nueve hijos de su matrimonio con Magdalena Mata y su ambición era aumentar su docencia y, con ello, sus emolumentos, algo que consiguió en 1908 al hacerse también cargo de la cátedra de Patología Quirúrgica con sus clínicas.

Como pediatra fue director de "la gota de leche" salmantina y en 1905 ingresó por oposición en el Cuerpo de Inspectores Provinciales de Sanidad. Defendió la introducción de nuevas técnicas, como la radiología, y estuvo abierto a todos los adelantos científicos a través de sus viajes de estudio, de los que se han conservado testimonios por su correspondencia con su amigo Miguel de Unamuno (Fig. 5).

\subsection{Hidrólogo e institucionalizador}

Pinilla ingresó en el Cuerpo de Médicos-Directores de Baños en la oposición de 1887, convocada para cubrir trece plazas vacantes. Es interesante señalar que en ese tribunal estaban presentes Anastasio García López, Marcial Taboada de la Riva y Joaquín Eduardo Gurucharri, quienes, como hemos visto, jugaron un importante papel en otros momentos de su vida profesional.

Su primer destino fue en Caldes de Malavella, a los que se fueron sucediendo los balnearios de Arteixo (18881889), Caldes de Reis (1890), Calzadilla del Campo (18911893), Incio (1894), El Molar (1895), Fuente Caliente (Miranda de Ebro, 1901-1903), Zaldívar (1904), San Hilario Sacalm (1909,1910), Molinar de Carranza (1911), Ledesma (1908, 1914-1917), Sobrón y Soportilla (19181921), Alceda y Ontaneda (1924-1925) y Caldas de Oviedo (1926-1932). Los frecuentes cambios de balneario están acordes con su ascenso en el escalafón y, fundamentalmente, la elección de los de mayor concurrencia que, sólo con la plaza de Caldas de Oviedo, llegó a ser mayor de dos mil agüistas por temporada.

Fue miembro de la Sociedad Española de Hidrología Médica, en la que durante muchos años se encargó de la publicación de los Anales, y llegó a presidirla brevemente en 1930.

Sería muy difícil referir toda la labor que realizó en el ámbito de la Hidrología Médica, por lo que intentaremos destacar las que pudieron tener una mayor trascendencia. La investigación, como más adelante veremos, fue una de las prioridades de Pinilla, quien consideraba que sólo a través de ella se conseguiría un cambio en el balnearismo español. Desde los análisis de aguas a la clínica (especialmente en afecciones pediátricas) amplió las líneas de estudio sobre las aguas medicinales e incorporó la climatología, publicando en 1930 un manual de referencia. Su principal cometido fue dotar a la disciplina de un corpus teórico dirigido no sólo a los profesionales hidrólogos o a su alumnado, sino, fundamentalmente, a esos médicos generales a los que había que instruir en el uso terapéutico de las aguas mineromedicinales. Su Manual de Hidrología Médica, reeditado en múltiples ocasiones, o su Diccionario General Hidrológico son muestras de ello. Pero también buscó crear escuela con la formación de discípulos como el malogrado José García Viñals y Antonia Martínez Casado.

\subsection{Político y visionario}

Hijo de Tomás Rodríguez Pinilla, político desterrado a Portugal por su vinculación revolucionaria a "la septembrina" y Consejero de Estado y Subsecretario de Hacienda durante el Sexenio, Hipólito no sólo adoptó ambos apellidos paternos sino también su ideología republicana y liberal que lo llevó a militar finalmente en el Partido Reformista de Melquiades Álvarez y a formar parte de la Junta Nacional Reformista en 1914.

De ambiguo posicionamiento durante la dictadura de Primo de Rivera, Manuel Márquez consideraba -al darle la réplica en su investidura en 1924 como miembro de la Real Academia de Medicina- que había superado el 
radicalismo de sus preferencias políticas juveniles, pero la correspondencia con Unamuno lo muestran como un "no significado" que mantenía sus contactos con los sectores críticos con el régimen. Esto explica que en mayo de 1931 fuese nombrado por el Gobierno Provisional de la República como miembro "a título personal por sus especiales conocimientos científicos"2 del Consejo de Sanidad. Era el mismo año de su jubilación, lo que probablemente alentó que se decidiese a proponer los cambios más radicales que hubiese conocido hasta entonces la disciplina.

Llevaba, según sus propias palabras, un año sin relación profesional con los médicos de baños desde que dimitió como Presidente de la Sociedad, cuando, el cuatro de junio de 1932, presentó ante el Consejo de Sanidad una ponencia firmada también por Marañón, Mariscal y Mayoral para que formase parte del proyecto de una futura Ley de Sanidad (Fig. 6). En ella, la base tercera suprimía el Cuerpo de Médicos de Baños, mientras que la base sexta planteaba la creación de un Instituto de Hidrología y Climatología Médicas que sería "el plantel de los futuros especialistas o Médicos adscritos al ejercicio profesional de las localidades balnearias"3.

Pinilla, buen conocedor de la evolución de los balnearios en Europa, intentaba introducir cambios que permitiesen la implantación del modelo docente e investigador del instituto, pero con ello consiguió ser considerado un traidor, "un enemigo del pueblo", por sus colegas del Cuerpo y la Sociedad que vieron intereses personales en su postura. Una condena colectiva que lo condujo al ostracismo y a que tras su fallecimiento por un proceso cardiaco, el 29 de marzo de 1936, sólo la Casa Charra en Madrid lo homenajease sin la presencia de médicos de baños, hidrólogos, ni discípulos.

Compleja y controvertida, la actividad profesional -íntimamente ligada a la política- de Hipólito Rodríguez Pinilla supuso un punto de inflexión trascendente en la historia de la Hidrología Médica española que, pese a una guerra y una dictadura, permitió la pervivencia académica de una disciplina.

\section{REFERENCIAS BIBLIOGRÁFICAS}

Este artículo se basa en los siguientes trabajos propios previos en los que se abordan tanto el tema como el período y permitirán encontrar informaciones más detalladas y un amplio aparato crítico de notas y bibliografía de investigadores que han abordado la figura de Hipólito Rodríguez Pinilla y la cátedra de Hidrología Médica. En el artículo sólo se han hecho constar en notas a pie de página las citas textuales.

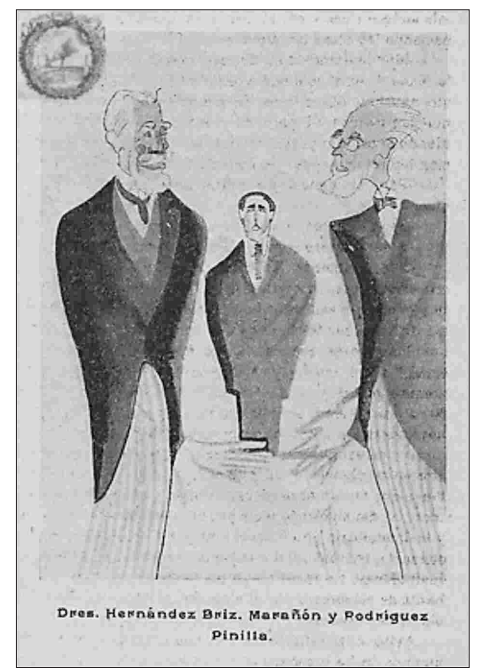

Fig. 6. Caricatura de Pinilla junto a Hernández Briz y Marañón (RANM).

- Rodríguez Sánchez, Juan Antonio. Hipólito Rodríguez Pinilla (18601936) y las estrategias institucionalizadoras de la Hidrología Médica en España. In: Cien años de la Cátedra de Hidrología Médica (Balnea. Serie de Monografias). Madrid: Universidad Complutense de Madrid, 2012, 13-47.

- Rodríguez Sánchez, Juan Antonio. Institucionalización de la Hidrología Médica en España. In: Maraver Eyzaguirre, Francisco (ed.). Establecimientos balnearios: historia, literatura y medicina. Madrid: Universidad Complutense de Madrid, 2006, 25-40.

- Rodríguez Sánchez, Juan Antonio. Ámbitos de poder de la Hidrología Médica española durante el franquismo. Estudos do Século XX. 2005, 5:159-177.

- Rodríguez. Sáncher, Juan Antonio. Antecedentes bistóricos: la(s) memoria(s) del agua. In: Baeza Rodríguez Caro, López Geta, Ramirez. Ortega (eds.) Las aguas minerales en España. Madrid: Instituto Geológico y Minero de España, 2001, 1-20.

- Rodríguez Sánchez, Juan Antonio. Los usos regeneracionistas de la simbología del agua: entre la decadencia balnearia y el moralismo kneippista. Dynamis. 1998, 18:107-126.

- Rodríguez, Sáncher, Juan Antonio. La creación de la Cátedra de Hidrología Médica (1912). In: Castellanos Guerrero, J.; Jiménez Lucena, I.; Ruiz Somavilla, M.J.; Gardeta Sabater, P. La medicina en el siglo XX. Estudios Históricos sobre Medicina, Sociedad y Estado. Málaga: Sociedad Española de Historia de la Medicina, 1998, 201-212. - Rodríguez Sáncher, Juan Antonio. La supresión del Cuerpo de Médicos de Baños (1932-1936). In: Castellanos Guerrero, J.; Jiménez Lucena, I.; Ruir Somavilla, M.J.; Gardeta Sabater, P. La medicina en el siglo XX. Estudios Históricos sobre Medicina, Sociedad y Estado. Málaga: Sociedad Española de Historia de la Medicina, 1998, 269-280. - Rodríguez Sánchez, Juan Antonio. José Salgado y Guillermo: el Premio en la Historia. Boletín de la Sociedad Española de Hidrología Médica (Cuarta Época). 1994, 9:105-111.

2 Ecos y noticias. Anales de la Sociedad Española de Hidrología Médica. 1931, 38:303-304.

3 Castells, R. Un acuerdo del Consejo de Sanidad. Anales de la Sociedad Española de Hidrología Médica. 1932, 39:90-93. 